\title{
Closed-Form Solution of a Peripherally Fixed Circular Membrane under Uniformly Distributed Transverse Loads and Deflection Restrictions
}

\author{
Teng-fei Wang $\mathbb{D}^{1},{ }^{1}$ Xiao-ting $\mathrm{He} \mathbb{D}^{1},{ }^{1,2}$ and Yang-hui Li $\mathbb{D}^{1}$ \\ ${ }^{1}$ School of Civil Engineering, Chongqing University, Chongqing 400045, China \\ ${ }^{2}$ Key Laboratory of New Technology for Construction of Cities in Mountain Area, Chongqing University, Ministry of Education, \\ Chongqing 400045, China
}

Correspondence should be addressed to Xiao-ting He; hexiaoting@cqu.edu.cn

Received 4 January 2018; Accepted 26 March 2018; Published 8 May 2018

Academic Editor: Fabrizio Greco

Copyright (c) 2018 Teng-fei Wang et al. This is an open access article distributed under the Creative Commons Attribution License, which permits unrestricted use, distribution, and reproduction in any medium, provided the original work is properly cited.

The problem of axisymmetric deformation of a peripherally fixed and uniformly loaded circular membrane under deflection restrictions (by a frictionless horizontal rigid plate) was analytically solved, where the assumption of constant membrane stress adopted in the existing work was given up, and a closed-form solution of this problem was presented for the first time. The numerical analysis shows that the closed-form solution presented here has higher calculation accuracy than the existing approximate solution.

\section{Introduction}

Elastic membrane structures and components are widely used in many fields [1-7]. The large deflection phenomena of membrane problem usually give rise to nonlinear differential equations [8-11]. These nonlinear equations generally present serious analytical difficulties when applied to boundaryvalue problems. Due to these somewhat intractable nonlinear equations, the analytical solutions of membrane problems are available in a few cases, but in practice they are often found to be necessary.

Hencky [12] originally dealt with the problem of axisymmetric deformation of the circular membrane fixed at the outer edge under the action of a uniformly distributed transverse loads and presented the power series solution of the problem, as shown in Figure 1, where $q$ is the uniformly distributed transverse loads. A calculation error in [12] was corrected by Chien [13] and Alekseev [14], respectively. This problem and its solution are usually called well-known Hencky problem and well-known Hencky solution for short, which are often referred to or cited in a number of related studies [15-22]. However, if we use a frictionless horizontal rigid plate to restrict the deflection of the membrane in the well-known Hencky problem, as shown in Figure 2, then such a problem will probably become somewhat complicated, where $r$ is the radial coordinate, $w$ is the transversal displacement, $b$ is the radius of the membrane contacting with the frictionless rigid plate, and $g$ is the gap between the frictionless rigid plate and the initially flat membrane. So, $\mathrm{Xu}$ and Liechti had to use the following four assumptions to deal with this problem [23]: (1) the membrane has negligible flexural rigidity and only membrane stresses are considered; (2) the slope angle $\theta$ of membrane is so small that the condition $\sin \theta \approx \tan \theta$ could approximately hold, that is, the socalled small-rotation-angle assumption; (3) a constant radial stress is assumed, that is, the radial stress in the membrane under loads has nothing to do with the radial coordinate; (4) the contact between the membrane and the rigid plate is frictionless. Obviously, assumption (3) above seems to be too harsh, and, in fact, it can be given up, as will be seen later.

In the next section, the problem shown in Figure 2 was analytically solved, where the assumption of constant membrane stress adopted in the existing work was given up, and the closed-form solution of this problem was presented. In Section 3, based on numerical calculations, the reliability of the presented closed-form solution is verified and the beneficial effect of giving up the assumption of constant 


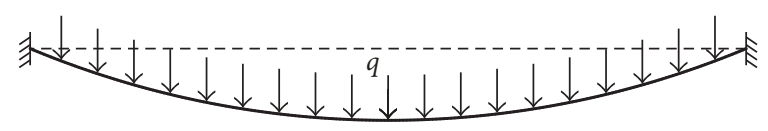

FIGURE 1: Sketch of the well-known Hencky problem.

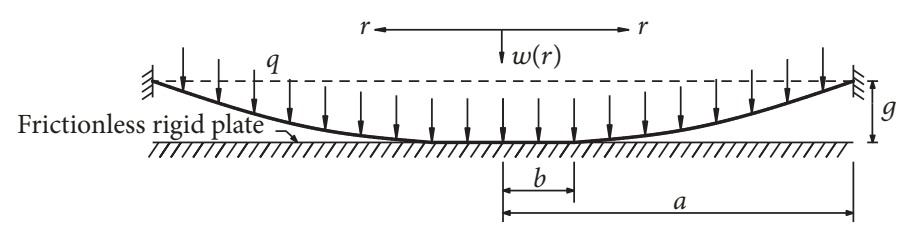

FIGURE 2: Sketch of confined deformation of the uniformly loaded circular membrane.

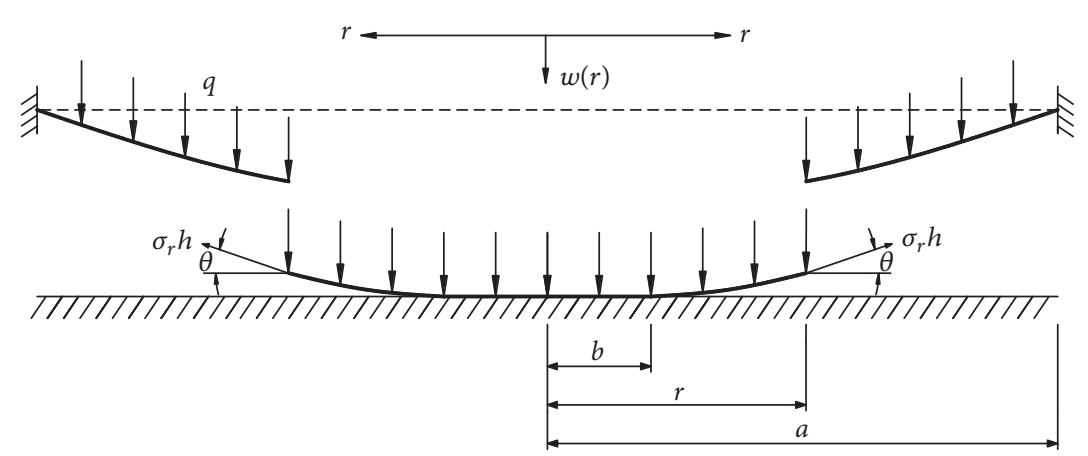

FIGURE 3: The equilibrium diagram of the central portion $(b \leq r \leq a)$ of the circular membrane.

membrane stress was discussed. Section 4 is the concluding remarks.

\section{Membrane Equation and Its Solution}

Suppose that, an initially flat, linearly elastic, rotationally symmetric, taut circular membrane with Young's modulus of elasticity $E$, Poisson's ratio $v$, thickness $h$, and radius $a$ is fixed at the outer edge and uniformly distributed transverse loads $q$ are quasi-statically applied onto the membrane surface (as shown in Figure 1, the well-known Hencky problem), and the applied loads $q$ especially have reached a value large enough so that the deflected membrane has a contact with a frictionless rigid plate being parallel to the initially flat membrane (as shown in Figure 2). Such a problem can be viewed as consisting of the two local problems in the central portion of $0<r \leq b$ and in the annular portion of $b \leq r \leq a$, which are connected by the continuity conditions at $r=b$, where the problem in $0<r \leq b$ may be simplified as a plane tension or compression problem of membrane, while the problem in $b \leq r \leq a$ is still a problem of membrane deflection. The following assumptions are here made in order to reach a closed-form solution: (1) during deformation the variation in thickness of membrane could be ignored, as is seen usually in membrane problems; (2) the so-called small-rotation-angle assumption reported in the existing literatures [18-20] is still adopted here, that is, the slope angle $\theta$ of membrane is so small that the condition $\sin \theta \approx \tan \theta$ could approximately hold; (3) the contact between the deflected membrane and the rigid plate is frictionless. Here, we give up the assumption of the constant radial stress reported in [23]. The continuous condition at $r=b$ and the boundary conditions at $r=a$ will be applied during the process of solution. As for the case prior to the contact between the deflected membrane and the frictionless rigid plate, that is, the well-known Hencky problem, it has been dealt with and its solution may be found in $[12,17]$.

Let us consider firstly the problem in the annular portion $(b \leq r \leq a)$ and take a piece of the central portion of the annular membrane whose radius is $r$; with a view of studying the static problem of equilibrium of this membrane under the joint action of the uniformly distributed loads $q$, the membrane force $\sigma_{r} h$ acted on the boundary and the reaction force from the rigid plate, just as it is shown in Figure 3, where $\sigma_{r}$ is the radial stress and $\theta$ is the slope angle of membrane. Right here there are three vertical forces, that is, the total force $\pi r^{2} q$ (in which $b \leq r \leq a$ ) of the uniformly distributed loads $q$, the total reaction force $\pi b^{2} q$ from the rigid plate, and the total vertical force $2 \pi r \sigma_{r} h \sin \theta$ which is produced by the membrane force $\sigma_{r} h$.

The out-plane equilibrium equation is

$$
2 \pi r \sigma_{r} h \sin \theta=\left(\pi r^{2}-\pi b^{2}\right) q,
$$

where

$$
\sin \theta \cong \tan \theta=-\frac{d w}{d r} .
$$

Substituting (2) into (1), one has

$$
2 r \sigma_{r} h \frac{d w}{d r}=-\left(r^{2}-b^{2}\right) q .
$$


The in-plane equilibrium equation is

$$
\frac{d}{d r}\left(r \sigma_{r} h\right)-\sigma_{t} h=0
$$

where $\sigma_{t}$ is the circumferential stress. The relations of strain and displacement of the large deflection problem are

$$
\begin{aligned}
& e_{r}=\frac{d u}{d r}+\frac{1}{2}\left(\frac{d w}{d r}\right)^{2}, \\
& e_{t}=\frac{u}{r},
\end{aligned}
$$

where $e_{r}$ is the radial strain, $e_{t}$ is the circumferential strain, and $u$ is the radial displacement. The relations of stress and strain are

$$
\begin{gathered}
\sigma_{r} h=\frac{E h}{1-v^{2}}\left(e_{r}+v e_{t}\right), \\
\sigma_{t} h=\frac{E h}{1-v^{2}}\left(e_{t}+v e_{r}\right) .
\end{gathered}
$$

Substituting (5a) and (5b) into (6a) and (6b),

$$
\begin{aligned}
& \sigma_{r} h=\frac{E h}{1-v^{2}}\left[\frac{d u}{d r}+\frac{1}{2}\left(\frac{d w}{d r}\right)^{2}+v \frac{u}{r}\right], \\
& \sigma_{t} h=\frac{E h}{1-v^{2}}\left[\frac{u}{r}+v \frac{d u}{d r}+\frac{v}{2}\left(\frac{d w}{d r}\right)^{2}\right] .
\end{aligned}
$$

By means of (4), (7a), and (7b), one has

$$
\frac{u}{r}=\frac{1}{E h}\left(\sigma_{t} h-v \sigma_{r} h\right)=\frac{1}{E h}\left[\frac{d}{d r}\left(r \sigma_{r} h\right)-v \sigma_{r} h\right] .
$$

If we substitute the $u$ of (8) into (7a), the compatibility equation may be written as

$$
r \frac{d}{d r}\left[\frac{1}{r} \frac{d}{d r}\left(r^{2} \sigma_{r} h\right)\right]+\frac{E h}{2}\left(\frac{d w}{d r}\right)^{2}=0 .
$$

The detailed derivation from (4) to (9) may be obtained from any general theory of plates and shells. It is not necessary to discuss this problem here.

Obviously, in the central portion of $0 \leq r \leq b$, the membrane is always in a plane state of radial tensile or compression; in other words, it is in fact only a plane problem with a salient characteristic of $d w / d r=0$. After substituting $d w / d r=0$ into (5a) and (5b), it may be found that

$$
\begin{aligned}
& e_{r}=\frac{d u}{d r} \\
& e_{t}=\frac{u}{r} .
\end{aligned}
$$

Substituting (10a) and (10b) into (6a) and (6b),

$$
\begin{aligned}
& \sigma_{r}=\frac{E}{1-v^{2}}\left(\frac{d u}{d r}+v \frac{u}{r}\right), \\
& \sigma_{t}=\frac{E}{1-v^{2}}\left(\frac{u}{r}+v \frac{d u}{d r}\right) .
\end{aligned}
$$

Substituting (11a) and (11b) into (4),

$$
r \frac{d^{2} u}{d r^{2}}+\frac{d u}{d r}-\frac{u}{r}=0
$$

So, considering the conditions

$$
\begin{aligned}
& u=0 \quad \text { at } r=0, \\
& u=u(b) \quad \text { at } r=b,
\end{aligned}
$$

the solution of (12) may be written as

$$
\frac{u(r)}{r}=\frac{u(b)}{b} .
$$

Substituting (14) into (10a), (10b), (11a), and (11b), it may finally be found that

$$
\begin{aligned}
& e_{r}=e_{t}=\frac{u(b)}{b}, \quad \text { in } 0 \leq r \leq b, \\
& \sigma_{r}=\sigma_{t}=\frac{E}{1-v} \frac{u(b)}{b}, \quad \text { in } 0 \leq r \leq b .
\end{aligned}
$$

Let us introduce the following nondimensional variables:

$$
\begin{aligned}
Q & =\frac{q a^{4}}{E h^{4}}, \\
W & =\frac{w}{h}, \\
S_{r} & =\frac{\sigma_{r} a^{2}}{E h^{2}}, \\
S_{t} & =\frac{\sigma_{t} a^{2}}{E h^{2}}, \\
x & =\frac{r}{a}, \\
\alpha & =\frac{b}{a},
\end{aligned}
$$

and transform (3), (9), (4), and (8) into

$$
\begin{gathered}
Q\left(x^{2}-\alpha^{2}\right)=-2 x S_{r} \frac{d W}{d x}, \\
x^{2} \frac{d^{2} S_{r}}{d x^{2}}+3 x \frac{d S_{r}}{d x}+\frac{1}{2}\left(\frac{d W}{d x}\right)^{2}=0, \\
S_{t}=S_{r}+x \frac{d S_{r}}{d x}, \\
\frac{u}{r}=\frac{h^{2}}{a^{2}}\left[(1-\nu) S_{r}+x \frac{d S_{r}}{d x}\right] .
\end{gathered}
$$

The boundary conditions, under which (17) and (18) may be solved, are

$$
\begin{aligned}
& \frac{u}{r}=0, \quad \text { at } x=1, \\
& W=0, \quad \text { at } x=1,
\end{aligned}
$$




$$
\begin{aligned}
\left(\frac{u}{r}\right)_{B} & =\left(\frac{u}{r}\right)_{A}=\frac{u(b)}{b}, \quad \text { at } x=\alpha, \\
W & =\frac{g}{h}, \quad \text { at } x=\alpha, \\
\left(S_{r}\right)_{B} & =\left(S_{r}\right)_{A}=\frac{a^{2}}{(1-v) h^{2}} \frac{u(b)}{b}, \quad \text { at } x=\alpha,
\end{aligned}
$$

where the subscripts $A$ and $B$ denote the values of various variables on two sides of the interconnecting circle $(r=b)$. The side of region $(A)$ is under the plane state of radial tensile or compression, while the side of region $(B)$ is under the deflection state of membrane. Eliminating $d W / d x$ from (17) and (18), we obtain a differential equation which contains only $S_{r}$ :

$$
x^{2} \frac{d^{2} S_{r}}{d x^{2}}+3 x \frac{d S_{r}}{d x}+\frac{Q^{2}}{8} \frac{\left(x^{2}-\alpha^{2}\right)^{2}}{\left(x S_{r}\right)^{2}}=0 .
$$

Letting $\beta=(1+\alpha) / 2$ and expanding $S_{r}$ into the power series of $x-\beta$, one has

$$
S_{r}=Q^{2 / 3} \sum_{i=0}^{\infty} c_{i}(x-\beta)^{i} .
$$

After substituting (24) into (23), the coefficients $c_{i}(i=$ $2,3,4, \ldots)$ can be expressed into the polynomial of the undetermined constants $c_{0}$ and $c_{1}$, which are shown in Appendix A. Further, expanding $W$ into the power series of $x-\beta$,

$$
W=Q^{1 / 3} \sum_{i=0}^{\infty} d_{i}(x-\beta)^{i},
$$

and substituting (24) and (25) into (17), the coefficients $d_{i}(i=$ $1,2,3, \ldots)$ can also be expressed into the polynomial of $c_{0}$ and $c_{1}$, which are shown in Appendix B, while $d_{0}$ is left as another undetermined constant, except $c_{0}$ and $c_{1}$. With the given $\nu$ and $\alpha=b / a$ all the undetermined constants can be determined by applying the continuous condition at $r=a$, as follows.

From (20) and (24), (21a) and (22a) give

$$
\begin{aligned}
& (1-v) \sum_{i=0}^{\infty} c_{i}(1-\beta)^{i}+\sum_{i=1}^{\infty} i c_{i}(1-\beta)^{i-1}=0 \\
& Q^{2 / 3} \frac{h^{2}}{a^{2}}\left[(1-\nu) \sum_{i=0}^{\infty} c_{i}(\alpha-\beta)^{i}+\alpha \sum_{i=1}^{\infty} i c_{i}(\alpha-\beta)^{i-1}\right] \\
& =\frac{u(b)}{b} .
\end{aligned}
$$

From (25), (21b) and (22b) give

$$
\begin{aligned}
& Q^{1 / 3} \sum_{i=0}^{\infty} d_{i}(1-\beta)^{i}=0, \\
& Q^{1 / 3} \sum_{i=0}^{\infty} d_{i}(\alpha-\beta)^{i}=\frac{g}{h} .
\end{aligned}
$$

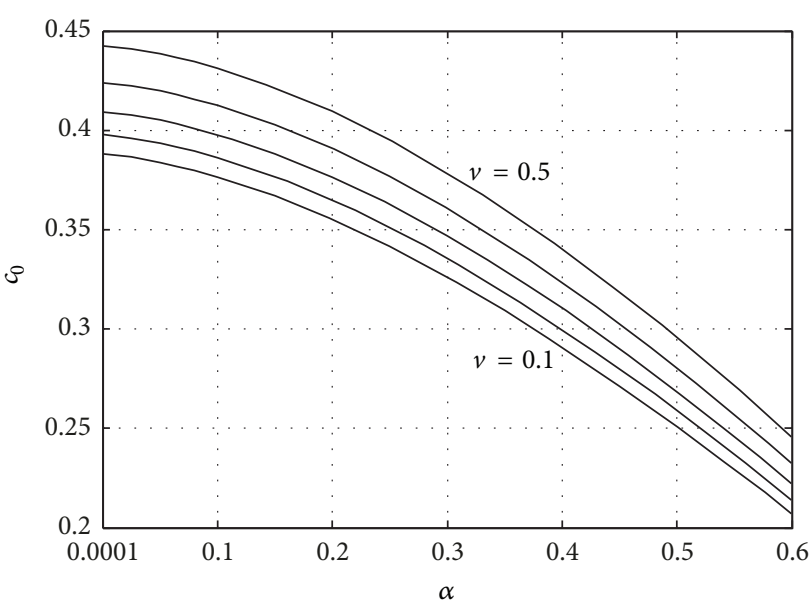

FIgURE 4: Variation of $c_{0}$ with $\alpha$.

From (24), (22c) gives

$$
Q^{2 / 3} \sum_{i=0}^{\infty} c_{i}(\alpha-\beta)^{i}=\frac{a^{2}}{(1-v) h^{2}} \frac{u(b)}{b} .
$$

Eliminating the $u(b)$ from (27) and (30), one has

$$
\sum_{i=1}^{\infty} i c_{i}(\alpha-\beta)^{i-1}=0 .
$$

Hence, for the given problem where $a, b, h, g, v$, and $E$ are known in advance, the undetermined constants $c_{0}$ and $c_{1}$ can be determined by (26) and (31), then the coefficients $c_{i}(i=2,3,4, \ldots)$ and $d_{i}(i=1,2,3, \ldots)$ can also be determined, and consequently the undetermined constant $d_{0}$ can be determined by (28). After this, the nondimensional variable $Q$ can be determined by (29), that is, the applied loads $q$, corresponding to this $b$, can be determined. Thus, the problem dealt with can be solved.

\section{Results and Discussions}

The undetermined constants $c_{0}, c_{1}$, and $d_{0}$ depend, in fact, on only $\nu$ and $\alpha=b / a$, as seen above. For the convenience of application, we here present the variation of the undetermined constants $c_{0}, c_{1}$, and $d_{0}$ with $\alpha$, as shown in Figures 4-6, where $v$ takes $0.1,0.2,0.3,0.4$, and 0.5 , respectively.

From the process of solution above we may see that, after giving up the assumption of the constant radial stress reported in [23], the solution to the problem dealt with becomes really complicated. Generally, the structural response is derived from an action of structure, but here the action, the applied loads $q$, has to be derived from the response, the contact radius $b$ of the membrane with the frictionless rigid plate.

For verifying the reliability of the closed-form solution presented here, we made a comparison between the solution presented here and the well-known Hencky solution [12, 17], based on the numerical example of a polyethylene 


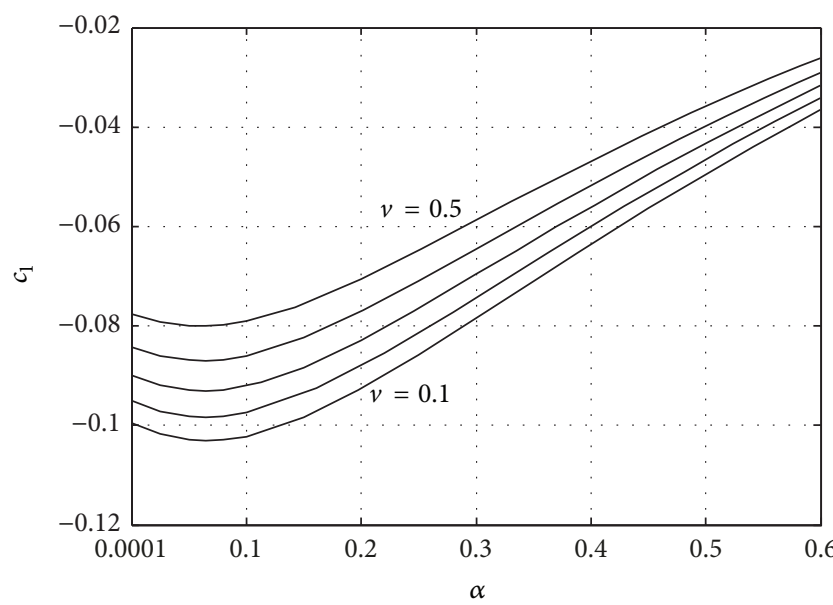

FIGURE 5: Variation of $c_{1}$ with $\alpha$.

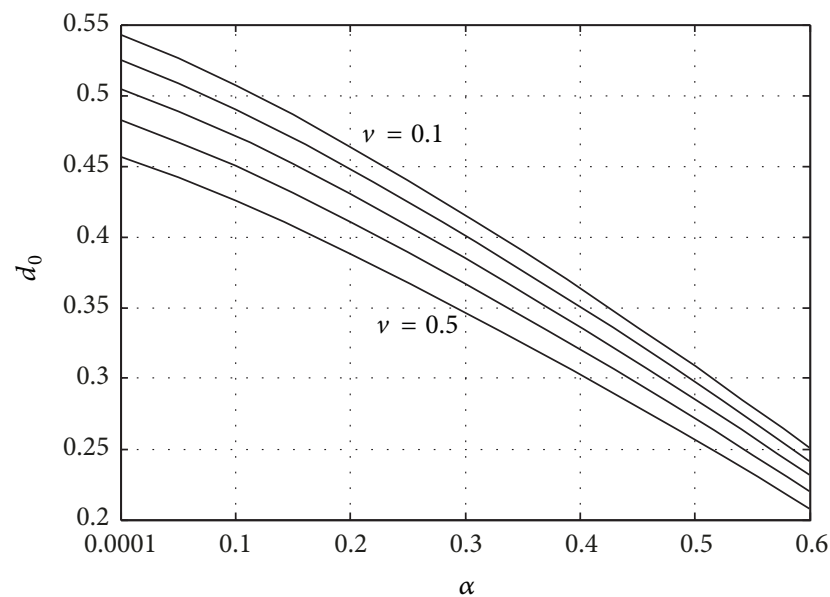

Figure 6: Variation of $d_{0}$ with $\alpha$.

terephthalate thin-film (considered in [23]) with $a=10 \mathrm{~mm}$, $h=0.003 \mathrm{~mm}, g=0.8 \mathrm{~mm}, E=4.65 \mathrm{GPa}$, and $v=$ 0.34 . When the contact radius $b$ is equal to $0.0001 \mathrm{~mm}$, the action, the applied loads $q$, is about $2.69 \mathrm{KPa}$. Figure 7 shows the variation of $w$ with $r$, where the solid line represents the result obtained by the solution presented here, and the dashed line represents the result obtained by the well-known Hencky solution. From Figure 7 it may be seen that the two profiles $w(r)$ are very close to each other; this shows that the closed-form solution presented here is, to some extent, reliable.

Further loading, when the contact radius $b$ is equal to $1 \mathrm{~mm}$, the applied loads $q$ are about $2.998 \mathrm{KPa}$. Figure 8 shows the variation of the radial stress $\sigma_{r}$ with the radial coordinate $r$, where the solid line represents the result obtained by the solution presented here, and the dashed dotted line represents the result obtained by the solution presented in [23] (the case without residual stress $\left.\sigma_{0}\right)$. This survey found a wide variation in the radial stresses $\sigma_{r}(r)$, which indicates that the assumption of the

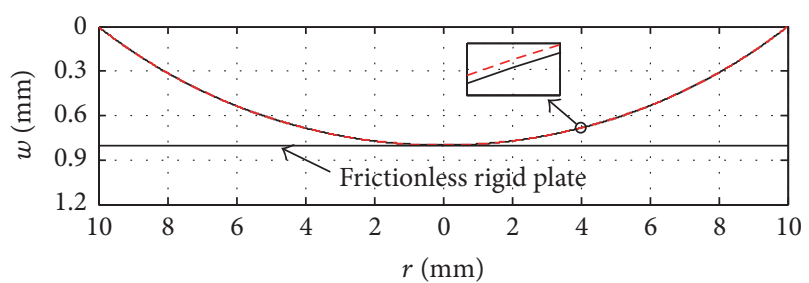

FIGURE 7: Variation of $w$ with $r$ when $q$ takes $2.69 \mathrm{KPa}$.

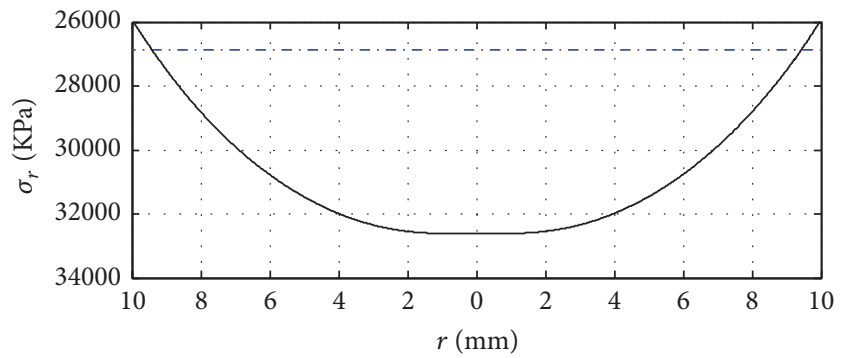

FIGURE 8: Variation of $\sigma_{r}$ with $r$ when $q$ takes $2.998 \mathrm{KPa}$.

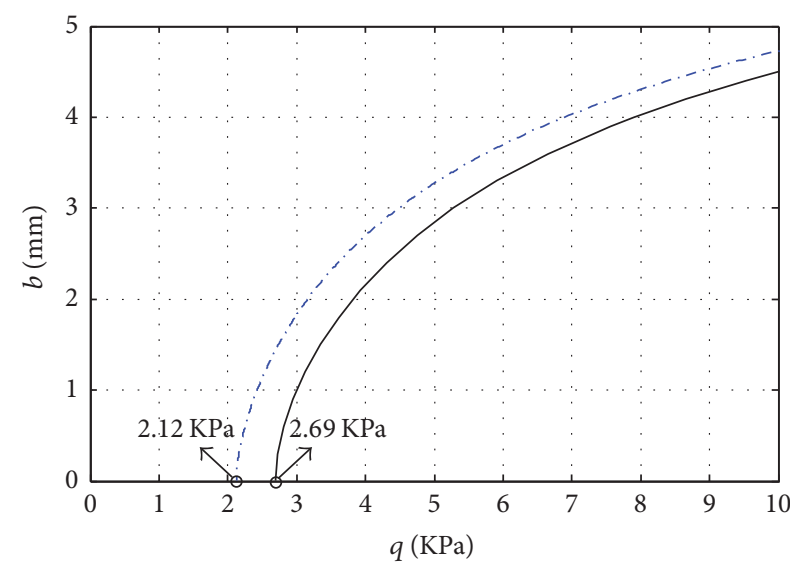

FIGURE 9: Variation of $b$ with $q$.

constant radial stress adopted in [23] should be given up.

Figure 9 shows the variation of the contact radius $b$ with the applied loads $q$, where the solid line represents the result obtained by the solution presented here, and the dashed dotted line represents the result obtained by the solution presented in [23] (the case without residual stress $\sigma_{0}$ ). As we can see from Figure 9, the dashed dotted line shows that the deflected membrane will start touching the frictionless rigid plate at $q \approx 2.12 \mathrm{KPa}$, while it starts touching the solid line at $q \approx 2.69 \mathrm{KPa}$. This difference is caused mainly by the assumption of the constant radial stress adopted in [23].

\section{Concluding Remarks}

In this study, the problem of axisymmetric deformation of a peripherally fixed circular membrane under uniformly 
distributed transverse loads was analytically dealt with under the condition of deflection restrictions, where the assumption of constant membrane stress adopted in [23] was given up, and the closed-form solution of this problem was presented for the first time. As far as well-known Hencky problem is concerned, the problem dealt with here can be understood as a new circular membrane problem under the combined loads, that is, the uniformly distributed transverse loads and the reaction force produced by the frictionless horizontal rigid plate.

The work presented here makes a significant and new contribution to thin-film mechanics. It ought to have significant implication in the mechanical characterization of film/substrate surface and interface and the interpretation of film/substrate delamination experiment; it could be incorporated especially into the study work reported in [23]. Further studies are expected to focus on giving up the socalled small-rotation-angle assumption adopted usually in membrane problems, in order to reach a closed-form solution with higher calculation accuracy and wider application scope.

\section{Appendix}

A. The Coefficients $c_{i}(i=2,3,4, \ldots)$

Letting $e=\beta^{2}-\alpha^{2}$, then the coefficients $c_{i}(i=2,3,4, \ldots)$ may be written as

$$
\begin{aligned}
c_{2} & =-\frac{1}{16} \frac{1}{\beta^{4} c_{0}^{2}}\left(24 \beta^{3} c_{0}^{2} c_{1}+e^{2}\right) \\
c_{3} & =\frac{1}{48} \frac{1}{\beta^{5} c_{0}^{3}}\left(96 \beta^{3} c_{0}^{3} c_{1}-4 \beta^{2} e c_{0}+2 \beta e^{2} c_{1}+7 e^{2} c_{0}\right) \\
c_{4} & =-\frac{1}{768} \frac{1}{\beta^{8} c_{0}^{5}}\left(1920 \beta^{5} c_{0}^{5} c_{1}+32 \beta^{6} c_{0}^{3}-64 \beta^{5} e c_{0}^{2} c_{1}\right. \\
& +24 \beta^{4} e^{2} c_{0} c_{1}^{2}-160 \beta^{4} e c_{0}^{3}+112 \beta^{3} e^{2} c_{0}^{2} c_{1} \\
& \left.+188 \beta^{2} e^{2} c_{0}^{3}+e^{4}\right) \\
c_{5} & =\frac{1}{3840} \frac{1}{\beta^{9} c_{0}^{6}}\left(11520 \beta^{5} c_{0}^{6} c_{1}+192 \beta^{7} c_{0}^{3} c_{1}\right. \\
& -288 \beta^{6} e c_{0}^{2} c_{1}^{2}+96 \beta^{5} e^{2} c_{0} c_{1}^{3}+384 \beta^{6} c_{0}^{4} \\
& -1152 \beta^{5} e c_{0}^{3} c_{1}+576 \beta^{4} e^{2} c_{0}^{2} c_{1}^{2}-1392 \beta^{4} e c_{0}^{4} \\
& +1272 \beta^{3} e^{2} c_{0}^{3} c_{1}+1368 \beta^{2} e^{2} c_{0}^{4}-16 \beta^{2} e^{3} c_{0} \\
& \left.+11 \beta e^{4} c_{1}+22 e^{4} c_{0}\right) \\
c_{6} & =-\frac{1}{184320} \frac{1}{\beta^{12} c_{0}^{8}}\left(9216 \beta^{10} c_{0}^{4} c_{1}^{2}-12288 \beta^{9} e c_{0}^{3} c_{1}^{3} e^{2} c_{0}^{2} c_{1}^{4}+645120 \beta^{7} c_{0}^{8} c_{1}+32256 \beta^{9} c_{0}^{5} c_{1}\right. \\
& +36 \beta^{8} e c_{0}^{4} c_{1}^{2}+28416 \beta^{7} e^{2} c_{0}^{3} c_{1}^{3}+31488 \beta^{8} c_{0}^{6}
\end{aligned}
$$

$$
\begin{aligned}
& -127488 \beta^{7} e c_{0}^{5} c_{1}+81216 \beta^{6} e^{2} c_{0}^{4} c_{1}^{2}-99456 \beta^{6} e c_{0}^{6} \\
& +113472 \beta^{5} e^{2} c_{0}^{5} c_{1}+88128 \beta^{4} e^{2} c_{0}^{6}+960 \beta^{6} e^{2} c_{0}^{3} \\
& -1920 \beta^{5} e^{3} c_{0}^{2} c_{1}+816 \beta^{4} e^{4} c_{0} c_{1}^{2}-3456 \beta^{4} e^{3} c_{0}^{3} \\
& \left.+3000 \beta^{3} e^{4} c_{0}^{2} c_{1}+2856 \beta^{2} e^{4} c_{0}^{3}+11 e^{6}\right) \\
& c_{7}=\frac{1}{1290240 \beta^{13} c_{0}^{9}}\left(507 e^{6} c_{0}+1315008 e^{2} \beta^{6} c_{0}^{5} c_{1}^{2}\right. \\
& +42744 e^{4} \beta^{2} c_{0}^{4}-66432 e^{3} \beta^{4} c_{0}^{4}+30528 e^{2} \beta^{6} c_{0}^{4} \\
& -3840 e \beta^{8} c_{0}^{4}+785664 e^{2} \beta^{4} c_{0}^{7}-949248 e \beta^{6} c_{0}^{7} \\
& +324864 \beta^{8} c_{0}^{7}+292 e^{6} \beta c_{1}+1310976 e^{2} \beta^{5} c_{0}^{6} c_{1} \\
& -77184 e^{3} \beta^{5} c_{0}^{3} c_{1}+18624 e^{2} \beta^{7} c_{0}^{3} c_{1} \\
& +61440 \beta^{11} c_{0}^{4} c_{1}^{3}-1672704 e \beta^{7} c_{0}^{6} c_{1} \\
& +511488 \beta^{9} c_{0}^{6} c_{1}-372 e^{5} \beta^{2} c_{0}+40080 e^{4} \beta^{4} c_{0}^{2} c_{1}^{2} \\
& -22656 e^{3} \beta^{6} c_{0}^{2} c_{1}^{2}+5160960 \beta^{7} c_{0}^{9} c_{1} \\
& -1375488 e \beta^{8} c_{0}^{5} c_{1}^{2}+304128 \beta^{10} c_{0}^{5} c_{1}^{2} \\
& +7632 e^{4} \beta^{5} c_{0} c_{1}^{3}+722688 e^{2} \beta^{7} c_{0}^{4} c_{1}^{3} \\
& -528384 e \beta^{9} c_{0}^{4} c_{1}^{3}+70584 e^{4} \beta^{3} c_{0}^{3} c_{1} \\
& +203520 e^{2} \beta^{8} c_{0}^{3} c_{1}^{4}-76800 e \beta^{10} c_{0}^{3} c_{1}^{4} \\
& \left.+23040 e^{2} \beta^{9} c_{0}^{2} c_{1}^{5}\right) \\
& c_{8}=-\frac{1}{20643840 \beta^{16} c_{0}^{11}}\left(73 e^{8}+14045184 \beta^{12} c_{0}^{7} c_{1}^{2}\right. \\
& -39648 e^{5} \beta^{4} c_{0}^{3}+12096 e^{4} \beta^{6} c_{0}^{3}+1268112 e^{4} \beta^{4} c_{0}^{6} \\
& -2297856 e^{3} \beta^{6} c_{0}^{6}+1352448 e^{2} \beta^{8} c_{0}^{6} \\
& -282624 e \beta^{10} c_{0}^{6}+15432192 e^{2} \beta^{6} c_{0}^{9} \\
& -44393472 e \beta^{9} c_{0}^{8} c_{1}-19574784 e \beta^{8} c_{0}^{9} \\
& +7045632 \beta^{10} c_{0}^{9}+36912 e^{6} \beta^{3} c_{0}^{2} c_{1} \\
& +32200704 e^{2} \beta^{9} c_{0}^{6} c_{1}^{3}+2920128 e^{4} \beta^{5} c_{0}^{5} c_{1} \\
& -4179456 e^{3} \beta^{7} c_{0}^{5} c_{1}+1718784 e^{2} \beta^{9} c_{0}^{5} c_{1} \\
& -187392 e \beta^{11} c_{0}^{5} c_{1}+31984128 e^{2} \beta^{7} c_{0}^{8} c_{1} \\
& +15360 \beta^{12} c_{0}^{6}+15151104 \beta^{11} c_{0}^{8} c_{1} \\
& +92897280 \beta^{9} c_{0}^{11} c_{1}+10980 e^{6} \beta^{4} c_{0} c_{1}^{2} \\
& -2552832 e^{3} \beta^{8} c_{0}^{4} c_{1}^{2}-516096 e^{3} \beta^{9} c_{0}^{3} c_{1}^{3}
\end{aligned}
$$




$$
\begin{aligned}
& +2591424 e^{4} \beta^{6} c_{0}^{4} c_{1}^{2}+541440 e^{2} \beta^{10} c_{0}^{4} c_{1}^{2} \\
& +42056064 e^{2} \beta^{8} c_{0}^{7} c_{1}^{2}+30828 e^{6} \beta^{2} c_{0}^{3} \\
& -24288 e^{5} \beta^{5} c_{0}^{2} c_{1}-50817024 e \beta^{10} c_{0}^{7} c_{1}^{2} \\
& +1024128 e^{4} \beta^{7} c_{0}^{3} c_{1}^{3}+5898240 \beta^{13} c_{0}^{6} c_{1}^{3} \\
& +150912 e^{4} \beta^{8} c_{0}^{2} c_{1}^{4}-30523392 e \beta^{11} c_{0}^{6} c_{1}^{3} \\
& +14146560 e^{2} \beta^{10} c_{0}^{5} c_{1}^{4}-9216000 e \beta^{12} c_{0}^{5} c_{1}^{4} \\
& +921600 \beta^{14} c_{0}^{5} c_{1}^{4}+3317760 e^{2} \beta^{11} c_{0}^{4} c_{1}^{5} \\
& \left.-1105920 e \beta^{13} c_{0}^{4} c_{1}^{5}+322560 e^{2} \beta^{12} c_{0}^{3} c_{1}^{6}\right)
\end{aligned}
$$

\section{B. The Coefficients $d_{i}(i=1,2,3, \ldots)$}

Letting $e=\beta^{2}-\alpha^{2}$, then the coefficients $d_{i}(i=1,2,3, \ldots)$ may be written as

$$
\begin{aligned}
d_{1} & =-\frac{1}{2} \frac{e}{c_{0} \beta} \\
d_{2} & =-\frac{1}{4} \frac{1}{\beta^{2} c_{0}^{2}}\left(2 \beta^{2} c_{0}-\beta e c_{1}-e c_{0}\right) \\
d_{3} & =\frac{1}{96} \frac{1}{\beta^{5} c_{0}^{4}}\left(32 \beta^{5} c_{0}^{2} c_{1}-16 \beta^{4} e c_{0} c_{1}^{2}+16 \beta^{4} c_{0}^{3}\right. \\
& \left.-40 \beta^{3} e c_{0}^{2} c_{1}-16 \beta^{2} e c_{0}^{3}-e^{3}\right) \\
d_{4} & =-\frac{1}{192} \frac{1}{\beta^{6} c_{0}^{5}}\left(48 \beta^{6} c_{0}^{2} c_{1}^{2}-24 \beta^{5} e c_{0} c_{1}^{3}+96 \beta^{5} c_{0}^{3} c_{1}\right. \\
& -96 \beta^{4} e c_{0}^{2} c_{1}^{2}+24 \beta^{4} c_{0}^{4}-108 \beta^{3} e c_{0}^{3} c_{1}-24 \beta^{2} e c_{0}^{4} \\
& \left.+5 \beta^{2} e^{2} c_{0}-4 \beta e^{3} c_{1}-5 e^{3} c_{0}\right) \\
d_{5} & =\frac{1}{1920} \frac{1}{\beta^{9} c_{0}^{7}}\left(384 \beta^{9} c_{0}^{3} c_{1}^{3}-192 \beta^{8} e c_{0}^{2} c_{1}^{4}\right. \\
& +1344 \beta^{8} c_{0}^{4} c_{1}^{2}-1056 \beta^{7} e c_{0}^{3} c_{1}^{3}+1248 \beta^{7} c_{0}^{5} c_{1} \\
& -1968 \beta^{6} e c_{0}^{4} c_{1}^{2}+192 \beta^{6} c_{0}^{6}-1344 \beta^{5} e c_{0}^{5} c_{1} \\
& -192 \beta^{4} e c_{0}^{6}-40 \beta^{6} e c_{0}^{3}+112 \beta^{5} e^{2} c_{0}^{2} c_{1} \\
& -58 \beta^{4} e^{3} c_{0} c_{1}^{2}+124 \beta^{4} e^{2} c_{0}^{3}-152 \beta^{3} e^{3} c_{0}^{2} c_{1} \\
& \left.-87 \beta^{2} e^{3} c_{0}^{3}-e^{5}\right) \\
& +1920 \beta^{6} c_{0}^{7}-19200 \beta^{5} e c_{0}^{6} c_{1}+160 \beta^{8} c_{0}^{4} \\
d_{6} & =-\frac{1}{23040} \frac{1}{\beta^{10} c_{0}^{8}}\left(3840 \beta^{10} c_{0}^{3} c_{1}^{4}-1920 \beta^{9} e c_{0}^{2} c_{1}^{5} c_{0}^{4} c_{1}^{3}-13440 \beta^{8} e c_{0}^{3} c_{1}^{4}+31680 \beta^{8} c_{0}^{5} c_{1}^{2}\right. \\
& -35040 \beta^{7} e c_{0}^{4} c_{1}^{3}+18240 \beta^{7} c_{0}^{6} c_{1}-40800 \beta^{6} e c_{0}^{5} c_{1}^{2} \\
& +190.007
\end{aligned}
$$

$$
\begin{aligned}
& -1216 \beta^{7} e c_{0}^{3} c_{1}+2104 \beta^{6} e^{2} c_{0}^{2} c_{1}^{2}-888 \beta^{5} e^{3} c_{0} c_{1}^{3} \\
& -1920 \beta^{4} e c_{0}^{7}-1232 \beta^{6} e c_{0}^{4}+5056 \beta^{5} e^{2} c_{0}^{3} c_{1} \\
& -3588 \beta^{4} e^{3} c_{0}^{2} c_{1}^{2}+2596 \beta^{4} e^{2} c_{0}^{4}-4416 \beta^{3} e^{3} c_{0}^{3} c_{1} \\
& \left.-1554 \beta^{2} e^{3} c_{0}^{4}+48 \beta^{2} e^{4} c_{0}-43 \beta e^{5} c_{1}-56 e^{5} c_{0}\right) \\
d_{7} & =\frac{1}{1290240} \frac{1}{\beta^{13} c_{0}^{10}}\left(184320 \beta^{13} c_{0}^{4} c_{1}^{5}\right. \\
& -92160 \beta^{12} e c_{0}^{3} c_{1}^{6}+1198080 \beta^{12} c_{0}^{5} c_{1}^{4} \\
& -783360 \beta^{11} e c_{0}^{4} c_{1}^{5}+2856960 \beta^{11} c_{0}^{6} c_{1}^{3} \\
& -2626560 \beta^{10} e c_{0}^{5} c_{1}^{4}+2972160 \beta^{10} c_{0}^{7} c_{1}^{2} \\
& -4343040 \beta^{9} e c_{0}^{6} c_{1}^{3}+1198080 \beta^{9} c_{0}^{8} c_{1} \\
& -3571200 \beta^{8} e c_{0}^{7} c_{1}^{2}+24576 \beta^{11} c_{0}^{5} c_{1} \\
& -115968 \beta^{10} e c_{0}^{4} c_{1}^{2}+158976 \beta^{9} e^{2} c_{0}^{3} c_{1}^{3} \\
& -59328 \beta^{8} e^{3} c_{0}^{2} c_{1}^{4}+92160 \beta^{8} c_{0}^{9}-1244160 \beta^{7} e c_{0}^{8} c_{1} \\
& +22272 \beta^{10} c_{0}^{6}-259584 \beta^{9} e c_{0}^{5} c_{1}+600384 \beta^{8} e^{2} c_{0}^{4} c_{1}^{2} \\
& -325440 \beta^{7} e^{3} c_{0}^{3} c_{1}^{3}-92160 \beta^{6} e c_{0}^{9}-122496 \beta^{8} e c_{0}^{6} \\
& -5416 \beta^{4} e^{5} c_{0} c_{1}^{2}+12608 \beta^{4} e^{4} c_{0}^{3}-14440 \beta^{3} e^{5} c_{0}^{2} c_{1} \\
& +681984 \beta^{7} e^{2} c_{0}^{5} c_{1}-626208 \beta^{6} e^{3} c_{0}^{4} c_{1}^{2} \\
& +217440 \beta^{6} e^{2} c_{0}^{6}-484608 \beta^{5} e^{3} c_{0}^{5} c_{1} \\
- & \left.118656 \beta^{4} e^{3} c_{0}^{6}-43 e^{7}\right) \\
& -4288 \beta^{6} e^{3} c_{0}^{3}+10336 \beta^{5} e^{4} c_{0}^{2} c_{1}
\end{aligned}
$$$$
d_{8}=-\frac{1}{5160960 \beta^{14} c_{0}^{11}}\left(-322560 e \beta^{6} c_{0}^{10}\right.
$$$$
+5329152 e^{2} \beta^{7} c_{0}^{6} c_{1}-79216 e^{5} \beta^{2} c_{0}^{4}
$$$$
+143840 e^{4} \beta^{4} c_{0}^{4}+947 e^{6} \beta^{2} c_{0}-1182 e^{7} c_{0}
$$$$
+14400 e^{2} \beta^{8} c_{0}^{4}-611712 e^{3} \beta^{4} c_{0}^{7}+1191888 e^{2} \beta^{6} c_{0}^{7}
$$$$
-741120 e \beta^{8} c_{0}^{7}+155904 \beta^{10} c_{0}^{7}-81664 e^{3} \beta^{6} c_{0}^{4}
$$$$
+322560 \beta^{8} c_{0}^{10}-203272 e^{5} \beta^{3} c_{0}^{3} c_{1}
$$$$
-3225600 e \beta^{12} c_{0}^{4} c_{1}^{6}+248224 e^{4} \beta^{5} c_{0}^{3} c_{1}
$$$$
-70848 e^{3} \beta^{7} c_{0}^{3} c_{1}-3349056 e^{3} \beta^{5} c_{0}^{6} c_{1}
$$$$
-322560 e \beta^{13} c_{0}^{3} c_{1}^{7}+645120 \beta^{14} c_{0}^{4} c_{1}^{6}
$$$$
-2517504 e \beta^{9} c_{0}^{6} c_{1}+365568 \beta^{11} c_{0}^{6} c_{1}
$$$$
-5644800 e \beta^{7} c_{0}^{9} c_{1}+5483520 \beta^{9} c_{0}^{9} c_{1}
$$ 


$$
\begin{aligned}
& -159400 e^{5} \beta^{4} c_{0}^{2} c_{1}^{2}+98232 e^{4} \beta^{6} c_{0}^{2} c_{1}^{2} \\
& -6096192 e^{3} \beta^{6} c_{0}^{5} c_{1}^{2}+7421376 e^{2} \beta^{8} c_{0}^{5} c_{1}^{2} \\
& -28385280 e \beta^{10} c_{0}^{6} c_{1}^{4}-892 e^{7} \beta c_{1}+177408 \beta^{12} c_{0}^{5} c_{1}^{2} \\
& -20885760 e \beta^{8} c_{0}^{8} c_{1}^{2}+18144000 \beta^{10} c_{0}^{8} c_{1}^{2} \\
& -39204 e^{5} \beta^{5} c_{0} c_{1}^{3}-4976064 e^{3} \beta^{7} c_{0}^{4} c_{1}^{3} \\
& +4110336 e^{2} \beta^{9} c_{0}^{4} c_{1}^{3}-665856 e \beta^{11} c_{0}^{4} c_{1}^{3} \\
& -33586560 e \beta^{9} c_{0}^{7} c_{1}^{3}+24514560 \beta^{11} c_{0}^{7} c_{1}^{3} \\
& -1888128 e^{3} \beta^{8} c_{0}^{3} c_{1}^{4}+792000 e^{2} \beta^{10} c_{0}^{3} c_{1}^{4} \\
& -2371584 e \beta^{10} c_{0}^{5} c_{1}^{2}+16128000 \beta^{12} c_{0}^{6} c_{1}^{4} \\
& -271872 e^{3} \beta^{9} c_{0}^{2} c_{1}^{5}-13224960 e \beta^{11} c_{0}^{5} c_{1}^{5} \\
& \left.+5160960 \beta^{13} c_{0}^{5} c_{1}^{5}\right) .
\end{aligned}
$$

\section{Conflicts of Interest}

The authors declare that they have no conflicts of interest.

\section{Acknowledgments}

This work was funded by the National Natural Science Foundation of China (Grants nos. 11772072 and 11572061).

\section{References}

[1] B. Schultrich, "Modified propagation of ultrasonic surface waves by thin elastic or viscous films," Zeitschrift Für Angewandte Mathematik und Mechanik, vol. 65, no. 1, pp. 19-27, 1985.

[2] J. Y. Sun, J. L. Hu, X. T. He, and Z. L. Zheng, "A theoretical study of a clamped punch-loaded blister configuration: The quantitative relation of load and deflection," International Journal of Mechanical Sciences, vol. 52, no. 7, pp. 928-936, 2010.

[3] J. Y. Sun, J. L. Hu, Z. L. Zheng, X. T. He, and H. H. Geng, "A practical method for simultaneous determination of Poisson's ratio and Young's modulus of elasticity of thin films," Journal of Mechanical Science and Technology, vol. 25, no. 12, pp. 3165-3171, 2011.

[4] J. Y. Sun, J. L. Hu, X. T. He, Z. L. Zheng, and H. H. Geng, "A theoretical study of thin film delamination using clamped punch-loaded blister test: Energy release rate and closed-form solution," Journal of Adhesion Science and Technology, vol. 25, no. 16, pp. 2063-2080, 2011.

[5] C. J. Liu, Z. L. Zheng, X. T. He et al., "L-P perturbation solution of nonlinear free vibration of prestressed orthotropic membrane in large amplitude," Mathematical Problems in Engineering, vol. 2010, Article ID 561364, 17 pages, 2010.

[6] S. M. Hasheminejad and A. Ghaheri, "Exact solution for vibration analysis of an eccentric elliptical membrane," Zeitschrift Für
Angewandte Mathematik und Mechanik, vol. 95, no. 7, pp. 730741, 2015.

[7] V. A. Eremeyev and K. Naumenko, "A relationship between effective work of adhesion and peel force for thin hyperelastic films undergoing large deformation," Mechanics Research Communications, vol. 69, pp. 24-26, 2015.

[8] J. Wauer and R. H. Plaut, "Vibrations of an extensible, airinflated, cylindrical membrane," Zeitschrift Für Angewandte Mathematik und Mechanik, vol. 71, no. 3, pp. 191-192, 1991.

[9] H. Grabmuller, "Wrinkle-free solutions in the theory of annular elastic membranes," Zeitschrift Für Angewandte Mathematik und Physik, vol. 42, no. 5, pp. 783-805, 1991.

[10] Z. L. Zheng, C. J. Liu, X. T. He, and S. L. Chen, "Free vibration analysis of rectangular orthotropic membranes in large deflection," Mathematical Problems in Engineering, vol. 2009, Article ID 634362, 9 pages, 2009.

[11] A. M. Kolesnikov and L. M. Zubov, "Large bending deformations of a cylindrical membrane with internal pressure," Journal of Applied Mathematics and Mechanics, vol. 89, no. 4, pp. 288305, 2009.

[12] H. Hencky, "Über den Spannungszustand in kreisrunden Platten mit verschwindender Biegungssteifigkeit," Zeitschrift Für Mathematik und Physik, vol. 63, pp. 311-317, 1915.

[13] W. Z. Chien, "Asymptotic behavior of a thin clamped circular plate under uniform normal pressure at very large deflection," The Science Reports of National Tsinghua University, vol. 5, pp. 71-94, 1948.

[14] S. A. Alekseev, "Elastic circular membranes under the uniformly distributed loads," Engineer Corpus, vol. 14, pp. 196-198, 1953 (Russian).

[15] H. J. Weinitschke, "Endliche Deformationen elastischer Membranen," Zeitschrift Für Angewandte Mathematik und Mechanik, vol. 53, no. 12, pp. 89-90, 1973.

[16] A. M. Arthurs and J. Clegg, "On the solution of a boundary value problem for the nonlinear Föppl-Hencky equation," Zeitschrift Für Angewandte Mathematik und Mechanik, vol. 74, no. 7, pp. 281-284, 1994.

[17] J. Y. Sun, Y. Rong, X. T. He, X. W. Gao, and Z. L. Zheng, "Power series solution of circular membrane under uniformly distributed loads: Investigation into Hencky transformation," Structural Engineering and Mechanics, vol. 45, no. 5, pp. 631-641, 2013.

[18] J. Y. Sun, S. H. Qian, Y. M. Li, X. T. He, and Z. L. Zheng, "Theoretical study of adhesion energy measurement for film/substrate interface using pressurized blister test: Energy release rate," Measurement, vol. 46, no. 8, pp. 2278-2287, 2013.

[19] J. Y. Sun, S. H. Qian, Y. M. Li, X. T. He, and Z. L. Zheng, "Theoretical study on shaft-loaded blister test technique: Synchronous characterization of surface and interfacial mechanical properties," International Journal of Adhesion and Adhesives, vol. 51, pp. 128-139, 2014.

[20] J. Y. Sun, Y. S. Lian, Y. M. Li, X. T. He, and Z. L. Zheng, "Closedform solution of elastic circular membrane with initial stress under uniformly-distributed loads: extended Hencky solution," Zeitschrift Für Angewandte Mathematik und Mechanik, vol. 95, no. 11, pp. 1335-1341, 2015.

[21] Y. S. Lian, J. Y. Sun, Z. X. Yang, X. T. He, and Z. L. Zheng, "Closed-form solution of well-known Hencky problem without small-rotation-angle assumption," Zeitschrift Für Angewandte Mathematik und Mechanik, vol. 96, no. 12, pp. 1434-1441, 2016. 
[22] Y. S. Lian, X. T. He, G. H. Liu, J. Y. Sun, and Z. L. Zheng, "Application of perturbation idea to well-known Hencky problem: A perturbation solution without small-rotation-angle assumption," Mechanics Research Communications, vol. 83, pp. 32-46, 2017.

[23] D. Xu and K. M. Liechti, "Analytical and experimental study of a circular membrane in Hertzian contact with a rigid substrate," International Journal of Solids and Structures, vol. 47, no. 7-8, pp. 969-977, 2010. 


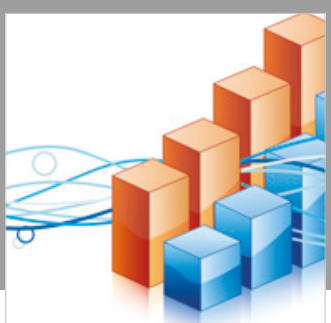

Advances in

Operations Research

\section{-n-m}
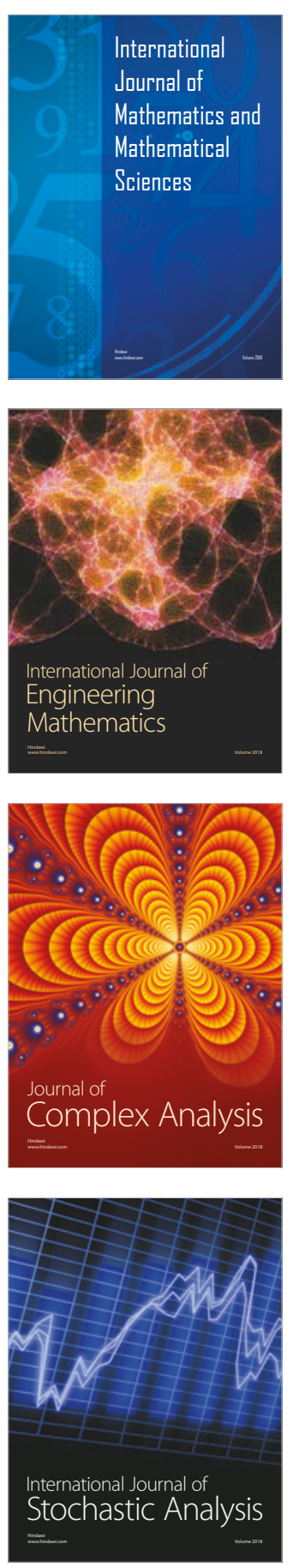
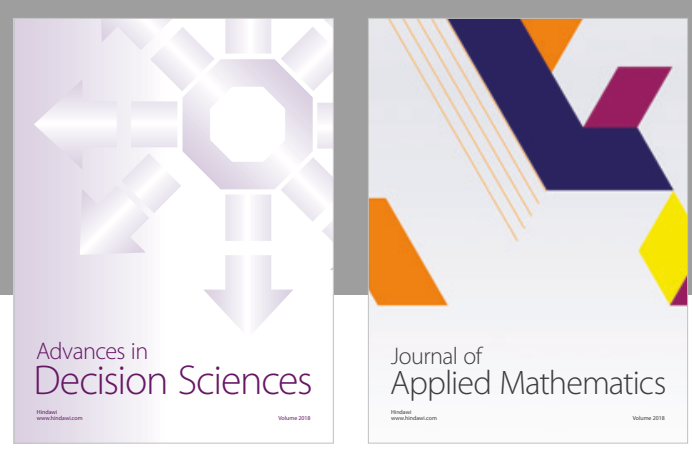

Journal of

Applied Mathematics
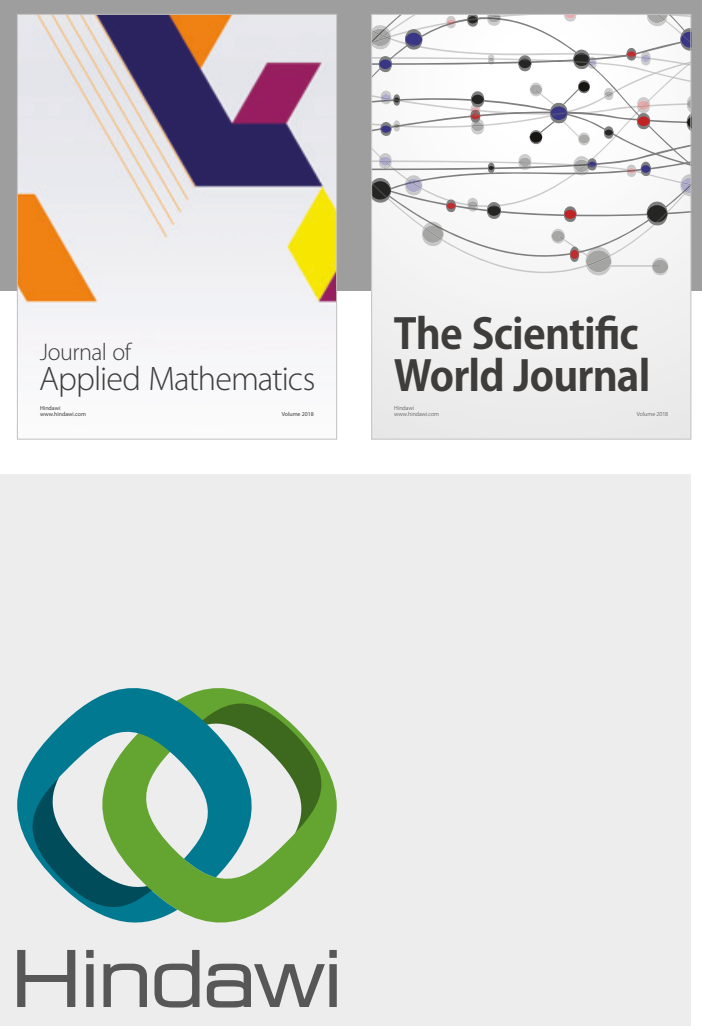

Submit your manuscripts at

www.hindawi.com

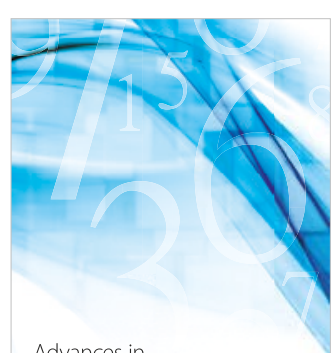

Advances in
Numerical Analysis
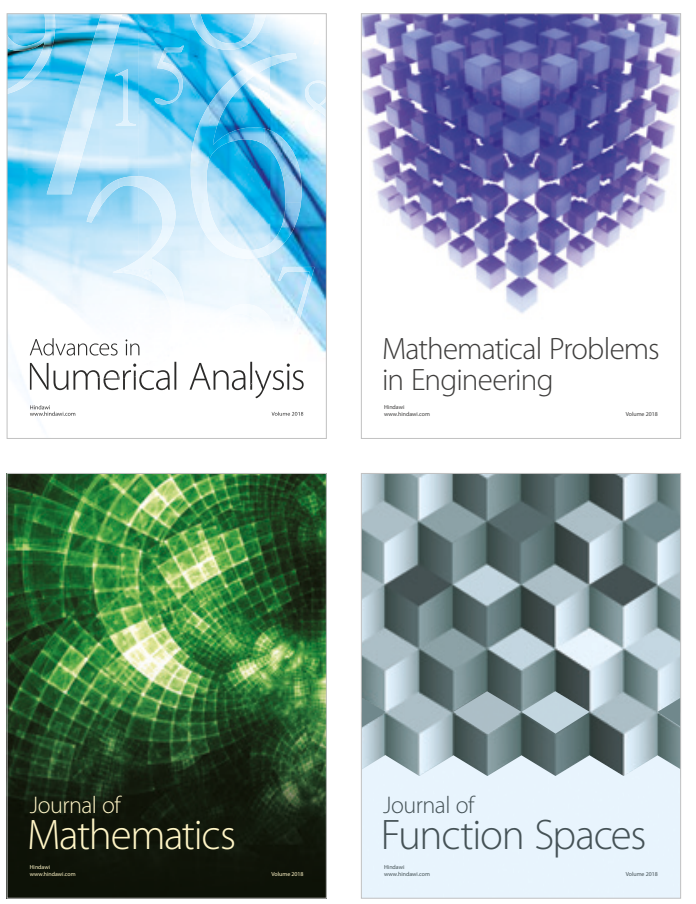

Mathematical Problems in Engineering

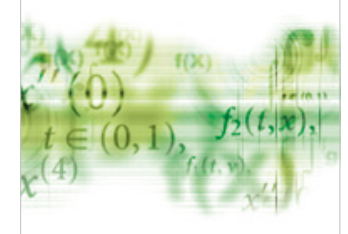

International Journal of

Differential Equations

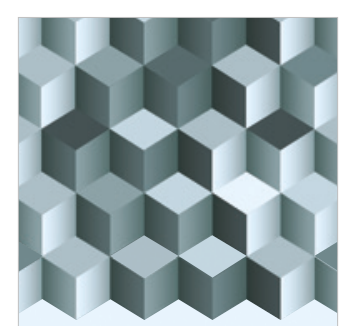

Journal of

Function Spaces

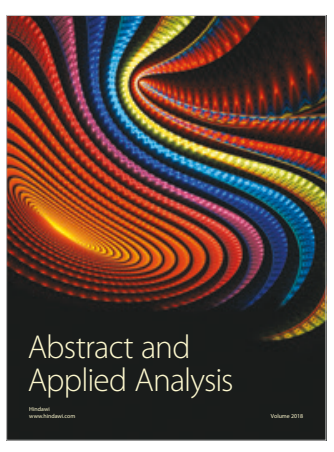

The Scientific

World Journal

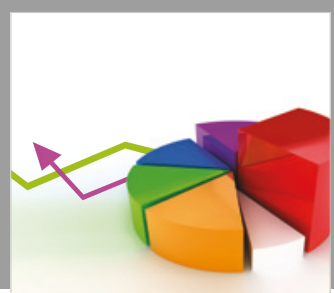

Journal of

Probability and Statistics
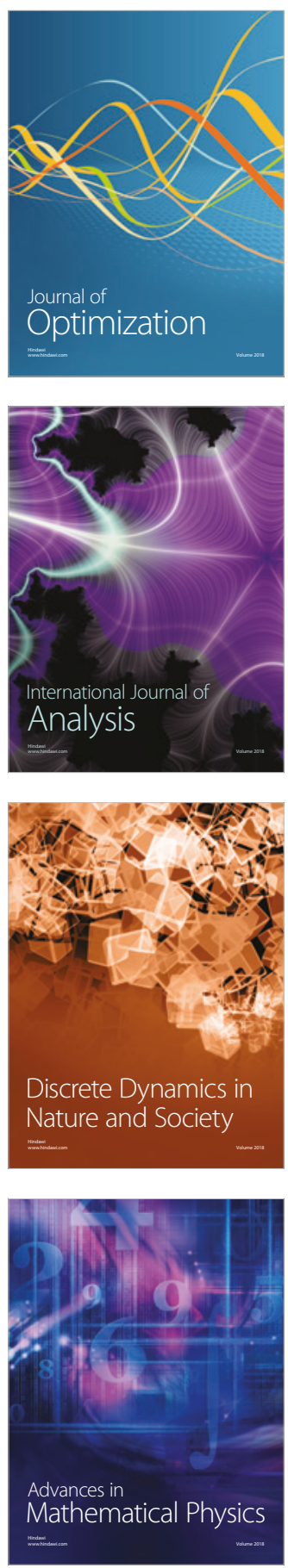